\title{
No gas source, no problem: proximity to pre-existing embolism and segmentation affect embolism spreading in angiosperm xylem by gas diffusion
}

\author{
Xinyi Guan ${ }^{1}$, Luciano Pereira ${ }^{1}$, Scott McAdam ${ }^{2}$, Kun-Fang Cao ${ }^{3}$, and Steven Jansen ${ }^{4}$ \\ ${ }^{1}$ Institute of Systematic Botany and Ecology, Ulm University, \\ ${ }^{2}$ Purdue University \\ ${ }^{3}$ State Key Laboratory for Conservation and Utilisation of Subtropical Agro-Bioresources, \\ Guangxi University \\ ${ }^{4}$ Institute of Systematic Botany and Ecology, Ulm University
}

November 17, 2020

\begin{abstract}
Embolism spreading in dehydrating angiosperm xylem is driven by gas movement between embolised and sap-filled conduits. Here, we examine how proximity to pre-existing embolism and hydraulic segmentation affect embolism propagation. Based on the optical method, we compared xylem embolism resistance between detached leaves and leaves attached to branches, and between intact leaves and leaves with cut minor veins for six species. Moreover, we directly compared the optical and pneumatic method on detached leaves. Embolism resistance of detached leaves was significantly lower than leaves attached to stems, except for two species with all vessels ending in their petioles. Cutting of minor veins showed limited embolism spreading in minor veins near the cuts prior to major veins. Moreover, there was strong agreement in embolism resistance between the optical and pneumatic method, with minor differences occurring during early stages of embolism formation. We conclude that embolism resistance may represent a relative trait, depending on the proximity and connectivity to pre-existing embolism as a gas source. Since embolism formation may not rely on a certain pressure difference threshold between functional and embolised conduits, we suggest that embolism is facilitated by pressure-driven gas diffusion, while hydraulic segmentation can prevent embolism propagation by reducing gas diffusion.
\end{abstract}

No gas source, no problem: proximity to pre-existing embolism and segmentation affect embolism spreading in angiosperm xylem by gas diffusion

Xinyi Guan ${ }^{1}$, Luciano Pereira ${ }^{1,2,3}$, Scott A. M. McAdam ${ }^{4}$, Kunfang Cao ${ }^{5,6}$, Steven Jansen ${ }^{1}$

1. Institute of Systematic Botany and Ecology, Ulm University, Albert-Einstein-Allee 11, 89081, Ulm, Germany

2. Laboratory of Plant Physiology "Coaracy M. Franco", Center R\&D in Ecophysiology and Biophysics, Agronomic Institute (IAC), Campinas, Brazil

3. Laboratory of Crop Physiology, Department of Plant Biology, Institute of Biology, P.O. Box 6109, University of Campinas (UNICAMP), Campinas 13083-970, Brazil

4. Purdue Center for Plant Biology, Department of Botany and Plant Pathology, Purdue University, 915 W. State St. West Lafayette, IN, 47907, USA. 
5. Plant Ecophysiology and Evolution Group, State Key Laboratory for Conservation and Utilisation of Subtropical Agro-Bioresources, Guangxi University, Nanning, Guangxi, 530004, China.

6. Guangxi Key Laboratory of Forest Ecology and Conservation, College of Forestry, Guangxi University, Nanning, Guangxi, 530004, China.

* Corresponding author:xinyi.guan@uni-ulm.de

Funding information: The German Research Foundation (DFG, project Nr. 410768178)

\section{Abstract}

Embolism spreading in dehydrating angiosperm xylem is driven by gas movement between embolised and sap-filled conduits. Here, we examine how proximity to pre-existing embolism and hydraulic segmentation affect embolism propagation. Based on the optical method, we compared xylem embolism resistance between detached leaves and leaves attached to branches, and between intact leaves and leaves with cut minor veins for six species. Moreover, we directly compared the optical and pneumatic method on detached leaves. Embolism resistance of detached leaves was significantly lower than leaves attached to stems, except for two species with all vessels ending in their petioles. Cutting of minor veins showed limited embolism spreading in minor veins near the cuts prior to major veins. Moreover, there was strong agreement in embolism resistance between the optical and pneumatic method, with minor differences occurring during early stages of embolism formation. We conclude that embolism resistance may represent a relative trait, depending on the proximity and connectivity to pre-existing embolism as a gas source. Since embolism formation may not rely on a certain pressure difference threshold between functional and embolised conduits, we suggest that embolism is facilitated by pressure-driven gas diffusion, while hydraulic segmentation can prevent embolism propagation by reducing gas diffusion.

\section{Key-words}

Bordered pits, embolism, vessel network, angiosperm xylem, drought stress, optical method, pneumatic method, gas diffusion

\section{Acknowledgements}

We thank Dr. H. Jochen Schenk (California State University, Fullerton) and Lucian Kaack (Ulm University) for valuable comments on an early version of this paper, Dr. Melvin Tyree (Zhejiang Normal University) for useful discussions about pneumatic experiments, two anonymous reviewers for helpful suggestions, and various colleagues from the botanical garden of Ulm University for assistance with sampling. XG, KC, and SJ acknowledge financial support by the German Research Foundation (DFG, project Nr. 410768178). SAMM acknowledges the Humboldt Foundation for a postdoctoral fellowship at Ulm University. The authors declare that there is no conflict of interest.

No gas source, no problem: proximity to pre-existing embolism and segmentation affect embolism spreading in angiosperm xylem by gas diffusion

Running title: Pre-exist embolism affects embolism spreading

Xinyi Guan ${ }^{1}$, Luciano Pereira ${ }^{1,2,3}$, Scott A. M. McAdam ${ }^{4}$, Kunfang Cao ${ }^{5,6}$, Steven Jansen ${ }^{1}$

1. Institute of Systematic Botany and Ecology, Ulm University, Albert-Einstein-Allee 11, 89081, Ulm, Germany

2. Laboratory of Plant Physiology "Coaracy M. Franco", Center R\&D in Ecophysiology and Biophysics, Agronomic Institute (IAC), Campinas, Brazil

3. Laboratory of Crop Physiology, Department of Plant Biology, Institute of Biology, P.O. Box 6109, University of Campinas (UNICAMP), Campinas 13083-970, Brazil 
4. Purdue Center for Plant Biology, Department of Botany and Plant Pathology, Purdue University, 915 W. State St. West Lafayette, IN, 47907, USA.

5. Plant Ecophysiology and Evolution Group, State Key Laboratory for Conservation and Utilisation of Subtropical Agro-Bioresources, Guangxi University, Nanning, Guangxi, 530004, China.

6. Guangxi Key Laboratory of Forest Ecology and Conservation, College of Forestry, Guangxi University, Nanning, Guangxi, 530004, China.

* Corresponding author: xinyi.guan@uni-ulm.de

\begin{abstract}
Embolism spreading in dehydrating angiosperm xylem is driven by gas movement between embolised and sap-filled conduits. Here, we examine how proximity to pre-existing embolism and hydraulic segmentation affect embolism propagation. Based on the optical method, we compared xylem embolism resistance between detached leaves and leaves attached to branches, and between intact leaves and leaves with cut minor veins for six species. Moreover, we directly compared the optical and pneumatic method on detached leaves. Embolism resistance of detached leaves was significantly lower than leaves attached to stems, except for two species with all vessels ending in their petioles. Cutting of minor veins showed limited embolism spreading in minor veins near the cuts prior to major veins. Moreover, there was strong agreement in embolism resistance between the optical and pneumatic method, with minor differences occurring during early stages of embolism formation. We conclude that embolism resistance may represent a relative trait, depending on the proximity and connectivity to pre-existing embolism as a gas source. Since embolism formation may not rely on a certain pressure difference threshold between functional and embolised conduits, we suggest that embolism is facilitated by pressure-driven gas diffusion, while hydraulic segmentation can prevent embolism propagation by reducing gas diffusion.
\end{abstract}

\title{
Key-words
}

Bordered pits, embolism, vessel network, angiosperm xylem, drought stress, optical method, pneumatic method, gas diffusion

\section{Author contributions}

XG performed the experiments, with help from LP and SAMM. SJ and XG conceived the original ideas. SJ with KC supervised the project. XG wrote the manuscript with support from SJ, and revisions from all authors.

\section{Introduction}

Xylem sap in plants is frequently transported under negative pressure (Dixon and Joly, 1895; Jansen and Schenk 2015). Under conditions of low soil water content and/or high transpiration rates, the tensile force of xylem sap may increase considerably, which could lead to interruption of water transport in tracheary elements by large gas bubbles (embolism). Understanding the frequency and mechanism behind embolism formation in plant species is important because the amount of embolised conduits may affect the transport of xylem sap, and therefore photosynthesis (Zhuet al. , 2013; Martin-StPaul et al. , 2017). There is strong and convincing evidence that drought-induced embolism formation occurs via bordered pits in cell walls of adjacent conduits (Zimmermann, 1983; Sperry \& Tyree, 1988; Jansen et al. , 2018; Kaack et al. , 2019). It has frequently been assumed that once the pressure difference between sap-filled conduits (under negative pressure) and embolised ones (under atmospheric pressure) exceeds a certain threshold, embolism spreads from an embolised conduit to a neighbouring one via the mesoporous pit membranes of bordered pits (Choat et al. , 2008; Tixier et al. , 2014; Wason et al. , 2018; Avila et al. , submitted). Although embolism spreading from previously embolised conduits has been well presented in many textbooks and papers (Zimmermann, 1983; Crombie et al. , 1985; Choat et al. , 2016; Lamarque et al. , 2018), various basic questions about this process remain unclear (Kaack et al. , 2019). Since gas movement across pit membranes may involve 
two different processes, namely mass flow and diffusion, we prefer the general term embolism spreading or propagation instead of air-seeding, which includes mass flow of gas across a pit membrane only.

An important question is whether spreading of embolism in xylem tissue is facilitated by the presence of pre-existing embolised conduits, because this would raise questions about the absolute value of embolism resistance, and whether or not the mechanism behind embolism spreading depends on a certain pressure difference. Pre-existing embolism could be embolised conduits from a previous growth ring or protoxylem (Kitinet al. 2004; Sano et al. , 2011; Hochberg et al. , 2016). Embolised conduits and local spreading of embolism could also occur when herbivores or xylem feeding insects damage conduits, or when a plant organ experiences other types of damage or die-back. Artificial embolism spreading may occur when xylem tissue has been cut open to take embolism resistance measurements, because when a transpiring plant is cut in air, the air-water meniscus is quickly pulled back into the conduit lumina until it stops at an interconduit pit membrane (Zimmermann, 1983). A widely used approach to evaluate embolism resistance is to measure the xylem water potential that corresponds to $50 \%$ loss of hydraulic conductance $\left(\Psi_{50}, \mathrm{MPa}\right)$, while the xylem water potential corresponding to $50 \%$ of the total amount of gas that can be extracted from a dehydrated xylem tissue has been suggested as an alternative, direct approach of embolism quantification (Pereiraet al. , 2016, 2020a; Zhang et al. , 2018; Oliveira et al. , 2019). Both experimental approaches rely on cut plant organs either due to the requirements to measure hydraulic conductivity, or gas diffusion kinetics of dehydrating samples. Moreover, dehydration of a cut branch or leaf can proceed much faster than dehydration of an intact plant (Cochard et al. , 2013; Hochberg et al. , 2017). Other methods, however, such as microCT observations and the optical method can be used to quantify embolism in a non-destructive way in intact plants (Brodribb et al. , 2016a, b, 2017; Choat et al. , 2016; Lamarque et al. , 2018).

The amount of embolism propagation could be limited by hydraulic segmentation, which represents a hydraulic constriction or bottleneck of the conduit network (Zimmermann, 1983; Tyree \& Ewer, 1991; Levionnoiset al. , 2020). In a broad sense, hydraulic segmentation has also been described as compartmentalisation, connectivity, sectoriality, or modularity, and may include narrow conduit dimensions and/or poorly interconnected conduits, which increase the resistance of the hydraulic pathway (Ellmore et al. , 2006; Loepfe et al. , 2007; Espino \& Schenk, 2009). It has frequently been suggested that these constrictions of the hydraulic pathway may cause a difference in embolism resistance, which is defined as vulnerability segmentation (Tyree and Ewers, 1991; Levionnois et al. , 2020). Leaves, for instance, are said to be less embolism resistant than stem xylem based on the vulnerability segmentation hypothesis, although results across a broad range of species are mixed and could partly be explained by the different methods used (Zhu et al. , 2016; Klepsch et al. , 2018; Skelton et al. , 2018; Levionnois et al. , 2020). Also, it has not been tested yet whether vulnerability segmentation is affected by pre-existing embolism.

In a few studies, considerable differences in embolism resistance have been reported between intact plants and xylem tissue. Cut-open stem xylem of Vitis vinifera and Laurus nobilis, for instance, were suggested to underestimate embolism resistance (Choat et al. , 2010, Torres-Ruiz et al. , 2015; Lamarque et al. , 2018). Removal of leaves in seedlings of the ring-porous speciesQuercus robur was found to result in artificial embolism formation in stem xylem based on microCT (Choat et al. , 2016). In a few species, however, the bench dehydration method, which is a widely applied method for hydraulic estimations of embolism resistance, was found to show no difference in embolism resistance between cut, dehydrating branches and dehydration of intact plants of Quercusand Populus (Bréda et al., 1993; Tyree et al. , 1992; Skelton et al. , 2018). While more species need to be studied to understand a possible artefact associated with embolism spreading from cut-open xylem, two explanations could be suggested for this observed discrepancy, i.e. why cut-open xylem may reduce embolism resistance and facilitate embolism propagation. First, it is possible that the cutting of conduits with sap under negative pressure introduces a cutting artefact, with embolism formation due to a sudden pressure drop (Wheeler et al., 2013; Torres-Ruiz et al., 2015). Second, embolism spreading could be prevented by hydraulic segmentation, which may occur at growth rings, nodes, and the transition between organs, such as leaf petioles or side branches (Sano et al. , 2011; Levionnois et al. , 2020). Indeed, vessels are known not to run completely randomly, but may end near nodes, side branches, stempetiole transitions, and between the vascular bundles of the petiole and major veins (Salleo et al. , 1984; 
André et al. , 1999, André, 2005, Wolfe et al. , 2016).

In this paper, we aim to test to what extent cut-open angiosperm xylem has an effect on embolism spreading in leaves across a diverse selection of six temperate species. In the first and the second experiment we investigate if embolism resistance of leaf xylem was affected by the proximity to cut-open conduits. We hypothesise that leaf xylem would be more vulnerable to embolism for detached leaves with a cut petiole compared to leaves attached to stem segments. However, not only the proximity to cut-open vessels, but also hydraulic segmentation at the stem-leaf, or the petiole-leaf blade transition could affect embolism spreading, and may prevent a potential artefact in measurements of embolism resistance near cut xylem tissue. We included species with both deciduous and marcescent leaves (i.e. species that retain dead leaves on the plant), and diffuse porous and ring-porous wood, because hydraulic segmentation can be associated with leaf phenology and vessel dimensions. If pit membranes in bordered pits of vessels and tracheids would function as safety valves that avoid the spreading of embolism from embolised to functional conduits, it is possible that embolism spreading is reduced by the number of interconduit endwalls and/or the connectivity between conduits (Kaack et al. , 2019; Johnsonet al. , 2020). Species that show hydraulic segmentation, may have safety valves composed of many tracheids and/or narrow, fibriform vessels.

Since drought-induced embolism is frequently reported to initiate in large vessels, while narrow and short vessels or tracheids embolise typically later at lower xylem water potentials (Scoffoni et al. , 2017; Klepsch et al. , 2018), we tested if embolism spreading in minor veins with narrow and short conduits would also be affected by the proximity to a cut-open vein. In this second experiment, we expect that narrow and short vessels near cut minor veins would embolise before embolism occurs in the large vessels of major veins, which would make narrow vessels seemingly more vulnerable than wide ones. We also predict that embolism spreading in minor veins near artificially induced cuts has a rather limited, local distribution due to the short dimensions of minor veins.

Finally, we aimed to test whether or not embolism spreading differs between intact vessels that are close to cut-open vessels in a petiole, and intact vessels in leaf veins that are located further away from cut-open xylem. For this reason, the Pneumatron and the optical vulnerability method were applied to the same detached leaf. If the pneumatic method would be subject to a potential artefact due to gas extraction from intact vessels that are neighbouring embolised, cut conduits, this method could systematically underestimate embolism resistance compared to the optical method. The pneumatic method, which estimates the changing gas volume in intact vessels during dehydration, showed a good agreement with hydraulic methods applied to stem segments (Pereira et al. , 2016, Zhang et al. , 2018). Direct comparison of the pneumatic and optical method to detached leaves ofEucalyptus camaldulensis suggested no significant difference for this species (Pereira et al. , 2020a), although a larger number of species should be tested to confirm this finding.

The three complementary sets of experiments will help us to address the question of whether embolism spreading in angiosperm xylem relies on a certain pressure difference threshold between embolised and functional conduits.

\section{Materials and methods}

\section{Study site and plant material}

The six angiosperm species studied included Betula pendula, Carpinus betulus, Fagus sylvatica, Liriodendron tulipifera, Prunus avium, and Quercus petraea. Samples were collected at Ulm University, Germany $\left(48^{\circ} 25^{\prime} 20.3^{\prime \prime} \mathrm{N}, 9^{\circ} 57^{\prime} 20.2^{\prime \prime} \mathrm{E}\right)$. Except for L. tulipifera, all species are common, native angiosperm trees to Ulm. Various individuals of L. tulipiferawere grown at the Botanical garden of Ulm University. Most species studied were diffuse porous, except for Q. petraea, which was ring-porous. Also, C. betulus and $Q$. petraea were considered as species with marcescent leaves during winter, with partial marcescent leaves in $F$. sylvatica. The remaining three species are deciduous. We selected four to five mature trees for each species. Healthy, mature and sun-exposed branches were sampled between June and September 2019 for experiment 1 and 3, and in May 2020 for experiment 2. 


\section{Vessel length measurements}

Maximum vessel length of stems and leaf petioles was determined by applying the air injection method (Greenidge, 1952). After connecting the basipetal part of a stem or petiole to a syringe, a ca. $150 \mathrm{kPa}$ pressure was applied, while the acropetal part was kept under water. Successive cuts at the proximal stem/petiole end were made under water with a razor blade at intervals of $10 \mathrm{~mm}$ for stems and $2 \mathrm{~mm}$ for leaves until the first continuous stream of air bubbles could be seen emerging from the cut end. The corresponding length was then measured and recorded as the maximum vessel length of a stem or leaf petiole $\left(\mathrm{MVL}_{\mathrm{stem}}\right.$ and $\mathrm{MVL}_{\text {petiole, }}$, respectively). At least six stems or leaves were taken for these measurements (Figure S1).

We measured the maximum vessel length at the stem-leaf transition for leaves attached to $0.5 \mathrm{~cm}$ long stem segments. This approach was applied to six samples per species based on the air injection method. The syringe was connected to the short stem sample, and the leaf was shortened until bubbles could be seen emerging from the cut end.

The vessel length distribution of leaf xylem was measured with a Pneumatron device (Pereira et al. , 2020b). Instead of injecting air (Cohen et al. , 2003; Wang et al. , 2014; Pan et al. , 2015), the amount of gas that could be sucked up via cut-open conduits allowed us to measure the air conductivity of open vessels while shortening leaf petioles. We then plotted the air conductivity of the cut-open vessels against the petiole length. The average vessel length was obtained by fitting the vessel length equation from Sperryet al. (2005) to our data.

We defined a segmentation index as the maximum vessel length at the petiole end divided by the petiole length. This index indicated to what extent the longest vessels from the petiole end run into the leaf blade. Values $<1$ indicated that vessels ended before the leaf blade started, while values $>1$ suggested that at least some vessels starting at the petiole end run directly into the midrib of the leaf blade.

\section{Xylem embolism resistance of leaves}

\section{Sample preparation}

All samples were collected before 9:00 in the morning. After cutting samples from a tree in air, large branches were immediately put in a water-filled bucket and a ca. $20 \mathrm{~cm}$ stem segment was cut from the stem base under water to avoid air entry as much as possible. The length of the remaining branch samples was between 80 and $160 \mathrm{~cm}$, which was much longer than the maximum vessel length $\left(\mathrm{MVL}_{\text {stem }}\right)$. Samples were covered up with a dark plastic bag, transferred to the lab within $10 \mathrm{~min}$, and then rehydrated for more than two hours.

\section{The optical method}

The optical method following Brodribb et al. (2016a, b) was applied to quantify the amount of leaf xylem embolism. A healthy, mature and undamaged leaf from each branch was fixed under a stereo microscope (Axio Zoom.V16, Zeiss, Jena, Germany) or in optical clamps (for more details, see http://www.opensourceov.org/). The surface area of the leaf, which was scanned for both the stereo microscope and clamps, was about 1

$\mathrm{cm}^{2}$. In general, images were taken every $5 \mathrm{~min}$, and the water potential was simultaneously monitored with a stem psychrometer at 10 min intervals (see below). Then, images were processed using the Fiji version of ImageJ (Schindelin et al. , 2012) and the "OpenSourceOV ImageJ Toolbox" was used to analyse the images. Image stacks were made to determine changes in the brightness of leaf veins, which was due to embolism formation. The Percentage of Embolised Pixels (PEP) was quantified over time at decreasing xylem water potentials, with $\mathrm{PEP}_{50}$ representing the xylem water potential corresponding to $50 \%$ of total embolised pixels (Brodribb et al. , 2016b).

\section{Pneumatic measurements}

An automatic Pneumatron apparatus was applied to measure gas diffusion kinetics of desiccating leaves (Pereira et al. , 2020a, Jansenet al. , In press). The principle of this apparatus was similar to the manual approach of Pereira et al. (2016) and Zhang et al. (2018), but with a much higher accuracy and temporal 
resolution. When samples for pneumatic measurements were prepared, the cut-open conduits embolised intentionally, which happened quickly by shaving the sample ends with a fresh razor blade in air.

Pneumatic measurements were taken every $15 \mathrm{~min}$. For this a $40 \mathrm{kPa}$ of absolute pressure was created with a vacuum pump, which extracted gas from a petiole end via a solenoid valve. The amount of gas extracted in a discharge tube with known volume could be measured with a pressure sensor. The vacuum pump reached $40 \mathrm{kPa}$ (i.e., the initial pressure $\mathrm{P}_{\mathrm{i}}$ ) within less than a second. Pressure data were recorded in a SD card at a time interval of $500 \mathrm{~ms}$. The final pressure $\left(\mathrm{P}_{\mathrm{f}}\right)$ was taken after $30 \mathrm{~s}$. According to the ideal gas law, the moles of air extracted from vessels $(\Delta \mathrm{n})$ could be calculated following the equation below:

$\Delta \mathrm{n}=\mathrm{n}_{\mathrm{f}}-\mathrm{n}_{\mathrm{i}}=\frac{(P f-P i) \times V}{\mathrm{RT}}($ Eqn 1$)$

where $n_{i}$ and $n_{f}$ represented the moles of air molecules at the initial and final pressure, $V$ was the fixed volume of the discharge tube $(1.1 \mathrm{~mL}), \mathrm{R}$ was the gas constant $\left(8.134 \mathrm{kPa} \mathrm{L} \mathrm{mol}^{-1} \mathrm{~K}^{-1}\right)$, and $\mathrm{T}$ was the room temperature in the lab (around $25^{\circ} \mathrm{C}$ ). Since a small $\mathrm{V}$ is needed to increase the measurement precision when a tiny amount of air is sucked from plant tissue, which is the case for detached leaves (Pereiraet al. , 2020a, Jansen et al. , In press), V was estimated as the maximum gas volume that could be extracted when leaves were fully dehydrated $\left(\mathrm{AD}_{\max }\right.$, see below) divided by 510.2 (Pereiraet al. , 2020a). The volume of air discharged $(\mathrm{AD}, \mu \mathrm{L})$ from vessels could then be calculated based on the ideal gas law, with $\mathrm{P}_{\text {atm }}$ being the atmospheric pressure: $\mathrm{AD}=10^{6} \times \Delta \mathrm{n} \mathrm{R} \mathrm{T/} \mathrm{P}_{\mathrm{atm}}($ Eqn 2),

Finally, the Percentage of Air Discharged (PAD, \%) was calculated:

$\mathrm{PAD}=100 \times\left(\mathrm{AD}-\mathrm{AD}_{\min }\right) /\left(\mathrm{AD}_{\max }-\mathrm{AD}_{\min }\right)(\mathrm{Eqn} 3)$

where $\mathrm{AD}_{\text {min }}$ was the minimum volume of air discharged when the leaf was well hydrated, and $\mathrm{AD}_{\max }$ was the maximum volume of air discharged when the leaf was strongly dehydrated.

Vulnerability curves were generated by plotting PAD or PEP against the corresponding leaf water potential $(\Psi)$, with a fitting by the following equation (Pammenter and Vander Willigen, 1998):

$\mathrm{PAD}$ or $\mathrm{PEP}=100 /\left(1+\exp (S / 25)\left(\Psi-\mathrm{P}_{50}\right)\right)($ Eqn 4$)$

$S$ represented the slope of the fitted curve, and $\mathrm{P}_{50}$ represented the water potential at $50 \%$ of air discharged, or $50 \%$ of the total embolised pixels of the leaf area scanned. Values of $\mathrm{P}_{12}$ (water potential at $12 \%$ of air discharged or embolised pixels) and $\mathrm{P}_{88}$ (water potential at $88 \%$ of air discharged or embolised pixels) were calculated following the equations by Domec and Gartner (2001):

$\mathrm{P}_{12}=2 /(S / 25)+\mathrm{P}_{50}($ Eqn 5$)$

$\mathrm{P}_{88}=-2 /(S / 25)+\mathrm{P}_{50}($ Eqn 6$)$

\section{Water potential measurements}

Psychrometers (PSY1, ICT International, Armidale, NSW, Australia) were applied to obtain water potential values from the leaves that were simultaneously measured with the optical and pneumatic method. Sand paper with a grit size of 400 was used to carefully remove a small area $\left(10 \mathrm{~mm}^{2}\right)$ of leaf cuticle under a stereomicroscope, while paying special attention not to abrade the vascular bundles and introduce air-entry into the xylem. Vaseline was used to seal the psychrometer chamber to the abraded surface of the leaf. Water potential measurements were taken every $10 \mathrm{~min}$, and the measurements were stopped when leaves were completely desiccated, or the water potential showed no further decrease over a long period. The duration of measurements was between one and two days.

At the beginning of each dehydration experiment, the leaf water potential dropped rapidly within one hour. After that, the water potential decreased slowly and steadily. To test the accuracy of the xylem water potential values taken with psychrometers, we applied two different approaches. Firstly, for leaves that were attached to long branches, we measured not only leaf xylem water potential, but also stem xylem water potential with another stem psychrometer (Fig. S2). This comparison provided a reasonably good match 
between both organs, although differences became more pronounced at high levels of dehydration for some species, with xylem water potential decreasing more quickly for leaves than stems. An exception was $F$. sylvatica, which had more negative xylem water potentials for leaves than stems when water potentials in leaves were less negative than -5 MPa. Secondly, water potential values of detached leaves that were measured with the Pneumatron and optical method were compared to a second set of leaves. Therefore, two leaves with a similar size were excised from the same branch at the same time. One leaf was attached to a psychrometer, while the other one was left to dry under similar conditions (same light intensity, temperature, and humidity). The water potential of the second leaf was measured with a pressure chamber (PMS Instrument Company, Albany, OR, USA) at an interval of 20 to 60 min to validate the accuracy of the stem psychrometer measurements during the first hours of dehydration. These tests revealed an overall good agreement between both methods for three species tested (C. betulus, F. sylvatica, and Q. petraea; Fig. S3).

\section{Experimental design}

We applied three different experiments to investigate the potential effect of cut-open xylem on embolism spreading.

Experiment 1: Comparison of detached leaves with leaves attached to a short and long stem segment

Embolism resistance of leaf xylem was measured using the optical method. For each of the six species studied, we considered three different types of samples: (1) a detached leaf with a cut petiole, (2) a single leaf and petiole connected to a short $(0.5 \mathrm{~cm})$ stem segment, regardless of the vessel length in stem xylem, and (3) a leaf attached to a long branch, which was at least two times the maximum vessel length as measured for stem xylem. Depending on the species, the branch length of the latter samples had a length of ca. 80 to 160 $\mathrm{cm}$. Four to five replicates were tested for each sample type. The dehydration time for each leaf or branch usually took 10 to 48 hours. L. tulipiferaand B. pendula were the slowest dehydrating species. Also, the dehydration speed of detached leaves was much faster than leaves attached to a long branch for $F$. sylvatica , P. avium, and C. betulus (Table $\mathrm{S} 1$ ).

Experiment 2: Comparison of intact leaves vs leaves with cut-open minor veins

To compare the potential impact of cut-open narrow vessels or tracheids in leaf xylem on embolism spreading, the optical method was applied on two adjacent leaves attached to a long branch, which was more than twice the maximum vessel length in stem xylem. This approach was applied to all six species. Two to three leaf pairs were tested for each species. Two mature, healthy and adjacent leaves were selected and placed under the stereomicroscope. In one leaf, we cut a few minor veins $\left(3^{\text {rd }}\right.$ or $4^{\text {th }}$ vein order $)$ with a razor blade. Four to six cuts were made and the length of each cut was about 1 to $2 \mathrm{~mm}$. The other leaf selected was kept intact. Cuts on leaves were made at the beginning of the dehydration procedure, and images were taken every 10 seconds to obtain a high temporal resolution of embolism formation within the first 10 minutes after making the cuts. Moreover, a transparent tape was applied to both leaf areas observed to avoid any potential difference in dehydration between the cut and intact leaf. After the first ten minutes of scanning the leaf, images were taken every five minutes.

Experiment 3: Comparison of the optical method with the Pneumatron

Both the optical method and the Pneumatron were applied to the same detached leaves to estimate embolism resistance of xylem tissue. The Pneumatron was connected to the cut leaf petiole, while the optical method was applied to the upper part of the leaf blades, as far away from the cut petiole as possible. In this way, we obtained the highest hydraulic distance between both methods, with the optical method focussing on the intact vessels in the upper leaf veins, and the Pneumatron measuring gas diffusion between the cut-open and first series of intact conduits of the petiole, and probably the lower part of the leaf blade. For each species, four replicates were tested.

\section{Data analysis}


Vulnerability curves were plotted and fitted using SigmaPlot 14 (Systat Software Inc., Erkrath, Germany). After testing data for normal distribution and homogeneity of variance, a one-way ANOVA was applied to test for significant difference between xylem embolism resistance of a detached leaf, and a leaf attached to a short or long branch. An independent t-test was applied to determine whether the optical and pneumatic method differed. Statistics were performed in SPSS 22 (IBM, Armonk, New York, USA), and all graphs were drawn in SigmaPlot.

\section{Results}

\section{Petiole vessel length measurements}

L. tulipifera and $Q$. petraea had the longest petiole vessels of the six species studied, with an average length of $8.19 \pm 1.47$ and $8.02 \pm 1.33 \mathrm{~cm}$ (mean \pm standard deviation), respectively. Despite having the longest petioles and the largest leaf surface area, vessels of $L$. tulipifera were always shorter than the petiole length, indicating that vessels at the base of the petiole ended well before the base of the lamina. Cut-open vessels in the petiole of $B$. pendula also ended before the base of the lamina. Petiole vessels of $Q$. petraea, however, were found to run from the petiole base up to the middle of the midrib. A similar observation was found for cut-open vessels in F. sylvatica and $P$. avium, which reached to half the length of the midrib. $C$. betulus had petiole xylem with vessels that were only slightly longer than the petiole (Table 1).

Q. petraea had the longest average vessel length in petiole xylem, with a value of $5.1 \pm 0.9 \mathrm{~cm}$, which was followed by L. tulipifera $(3.52 \pm 1.17 \mathrm{~cm})$. The mean vessel length in petioles of $B$. pendula, $F$. sylvatica, and $C$. betulus was shorter than $1 \mathrm{~cm}$. Since the petiole of $P$. avium had a pronounced notch, we were unable to obtain accurate vessel length measurements for this species with the Pneumatron, even when using glue or parafilm to avoid any leakage (Table 1).

\section{Experiment 1: Does embolism spreading depend on the proximity to cut conduits?}

The shape of the vulnerability curves obtained was consistently sigmoidal for the three sample types, i.e. detached leaves, leaves attached to a short stem segment, and leaves attached to a long stem segment (Figure 1). Embolism expansion in leaf veins started typically in major veins and proceeded to minor veins (Figure S4). We did not see different patterns in the progression of embolism formation among detached leaves and leaves attached to a short or long stem segment.

There was considerable variation in the $\mathrm{PEP}_{12}, \mathrm{PEP}_{50}$ and $\mathrm{PEP}_{88}$ values among the three types of samples for several species. Comparison of detached leaves with leaves attached to short stem segments showed a significant difference $(P<0.05)$ in $\mathrm{PEP}_{50}$ for $C$. betulus,$F$. sylvatica, $P$. avium and Q. petraea (Figure 1 , Table S2). Detached leaves of these species showed a ca. $1.5 \mathrm{MPa}$ less negative $\mathrm{PEP}_{50}$ value compared to $\mathrm{PEP}_{50}$ values of leaves on short stem segments. A minor difference in $\mathrm{PEP}_{50}$ with no significant difference was obtained for L. tulipifera and B. pendula (Figure 1, Table S2).

A positive, exponential correlation $\left(R^{2}=0.52, P<0.05\right)$ was found for the shift in embolism resistance between detached leaves and leaves attached to a short stem segment, and the segmentation index of leaf xylem (Figure 2). Leaves with a segmentation index $>1$ were strongly affected by the cut-open vessels at the petiole end, resulting in a shift in $\mathrm{PEP}_{12}, \mathrm{PEP}_{50}$ and $\mathrm{PEP}_{88}$ of $1 \mathrm{MPa}$ or more between detached leaves and leaves attached to stem segments. L. tulipifera and B. pendula, which had all vessels ending within their petiole, were clearly less affected by the proximity to cut xylem conduits compared to the four other species with vessels running from the petiole end into the midrib.

A significant difference in $\mathrm{PEP}_{12}$ and $\mathrm{PEP}_{50}$ was also found between leaves attached to a short branch, and those on a long branch for $F$. sylvatica. No significant difference in xylem embolism resistance was found between leaves on a short stem segment and leaves on a long stem piece for the other species studied, except for $\mathrm{PEP}_{88}$ values of $B$. pendula and $P$. avium. Any similarity or dissimilarity in $\mathrm{PEP}_{50}$ between the three types of samples was mostly reflected in $\mathrm{PEP}_{12}$ and $\mathrm{PEP}_{88}$ (Figure 1, Table S2).

Experiment 2: Does the proximity to cut conduits also affect embolism spreading in minor leaf 


\section{veins?}

The percentage of cumulative embolised xylem area in leaves with cut-open minor veins and intact leaves was plotted against time for the six species studied (Figure S5). For leaves with several artificial cuts in the $3^{\text {rd }}$ or $4^{\text {th }}$ order veins, no immediate embolism formation was detected after the cutting, except for the vessels that were cut open. In all six species, however, we observed embolism events in minor veins adjacent to the cuts during the first hours of desiccation (Figure 3, Figure S4). These embolism events in minor veins, with relatively narrow and short vessels, occurred prior to embolism formation in the wide and long vessels of major veins. Since the amount of embolised pixels near the cuts had a local effect only and was relatively small compared to the total amount of embolised pixels that could be detected in the entire leaf area, there was no large overall difference in embolism spreading between intact and cut leaves. Therefore, the embolism spreading patterns did not differ when the percentage of embolised pixels of the entire scanned leaf area was plotted against time. After artificially induced embolism events occurred near the cuts, embolism spreading followed in the major veins, and then spread to the minor veins in both intact and cut leaves (Figure 3).

\section{Experiment 3: Is the Pneumatron underestimating embolism resistance compared to the op-} tical method?

The optical method and Pneumatron were simultaneously applied to the same detached leaves for all six species. We were unable to obtain Pneumatron measurements for B. pendula, because the gas volume that could be extracted from the leaves was too small to meet the sensitivity requirements of the tube volume, even if we reduced the volume of the discharge tube to the minimum volume of $1.1 \mathrm{~mL}$.

Vulnerability curves based on the two methods were obtained for five species (Figure 4). The $\mathrm{PEP}_{12}, \mathrm{PEP}_{50}$ and $\mathrm{PEP}_{88}$ values of detached leaves obtained with the optical method in experiment 1 (Fig.1, Table S2) did not differ significantly from those obtained in experiment 3 (Table S3). A difference in embolism spreading from cut conduits to intact ones versus spreading between intact conduits would especially be reflected in $\mathrm{PAD}_{12}$ and $\mathrm{PEP}_{12}$ values. Indeed, average $\mathrm{PAD}_{12}$ values based on the Pneumatron were lower than the average $\mathrm{PEP}_{12}$ values based on the optical method for four species, except for $P$. avium. The difference between $\mathrm{PAD}_{12}$ and $\mathrm{PEP}_{12}$ was more than $0.6 \mathrm{MPa}$ for Q. petraea, F. sylvatica, and C. betulus, although these differences were not statistically significant. Also, there was considerable variation among the samples tested (Fig. 5a), with several samples showing a slightly more negative $\mathrm{PAD}_{12}$ value than $\mathrm{PEP}_{12}$ for $P$. avium and L. tulipifera .

Despite minor differences between $\mathrm{PEP}_{12}$ and $\mathrm{PAD}_{12}$, the vulnerability curves based on the optical and pneumatic method showed strikingly similar patterns for the five species tested, with a strong correlation between $\mathrm{PAD}_{50}$ and $\mathrm{PEP}_{50}$, and between $\mathrm{PAD}_{88}$ and $\mathrm{PEP}_{88}$ (Fig. 5b, c). When intraspecific differences in embolism resistance were found within a species, both methods matched each other very well (Figure 4, 5, Figure S6). Although curves based on the optical method had a relatively steeper slope compared to the pneumatic curves for F. sylvatica, $C$. betulus and $Q$. petraea, this difference was not significant. No significant difference was found between $\mathrm{PAD}_{50}$ and $\mathrm{PEP}_{50}$, and between $\mathrm{PAD}_{88}$ and $\mathrm{PEP}_{88}$. Nevertheless, $P$. avium showed a $0.67 \mathrm{MPa}$ difference $(P=0.287)$ between $\mathrm{PEP}_{50}$ and $\mathrm{PAD}_{50}$, and a $0.61 \mathrm{MPa}$ difference $(P$ $=0.454)$ between $\mathrm{PEP}_{88}$ and $\mathrm{PAD}_{88}$ (Table S3).

\section{Discussion}

Experiment 1 and 2 show that spreading of drought-induced embolism and thus embolism resistance can be strongly affected by the proximity of the xylem area studied to pre-existing embolism such as cut xylem tissue. The observation that embolism initiation $\left(\mathrm{PEP}_{12}\right)$ occurs over a $>1 \mathrm{MPa}$ range of xylem water potential for four out of six species studied challenges the assumption that embolism spreads once a certain threshold difference has been reached. While cut-open conduits may facilitate embolism spreading, hydraulic segmentation may limit this potential artefact. As predicted, embolism spreading in minor veins was also affected by the proximity to cut conduits, but showed a rather limited, local distribution without affecting the entire leaf. Also, we found no difference between the optical and pneumatic method for five species, despite considerable variation in the $\mathrm{P}_{12}$ values of both methods. The consequences and broader significance 
of these findings present some old questions and assumptions in a new light, and provides a novel gas diffusion hypothesis as a possible mechanism for embolism spreading.

\section{Embolism spreading depends on pre-existing embolism}

Since embolism spreading happens largely from one embolised conduit to a neighbouring one, as predicted by the air-seeding hypothesis (Zimmermann, 1983; Sperry and Tyree, 1988), the observation that embolism formation may depend on proximity to an existing gas source such as cut-open conduits or pre-existing embolised conduit is not surprising. Embolism formation appears to be unlikely if a conduit is not connected to a pre-existing embolism. Novel, de novo embolism formation has been observed in very few conduits that are not connected to embolised ones based on microCT (Brodersen et al. , 2013, Choatet al. , 2015, 2016), and embolism formation in seemingly isolated conduits could occasionally be observed in our experiments. However, the rather two-dimensional view associated with the optical method, its limited resolution to accurately detect narrow vessel ends (Oskolski and Jansen, 2009), and its shortcoming to detect pre-existing embolism, did not allow us to confirm that these conduits were completely disconnected from neighbouring gas sources.

If availability of a pre-existing gas source or embolised conduit is important, then where does the gas come from to induce embolism in intact xylem? It is possible that there is almost always an embolised conduit available, perhaps in primary xylem or in older xylem from an older growth ring. This would be an obvious gas source if functional, sap-filled conduits show any direct connection with these embolised conduits via bordered pits. However, these pre-existing gas sources may be limited due to poor connectivity or compartmentalisation of the hydraulic network (Kitin et al. , 2004; Morris et al. , 2016). Since vessels and tracheids do not share pits with non-conductive fibres (Sano et al. , 2011), it is unlikely that air entry from these cells or intercellular spaces will contribute to embolism formation in conduits.

\section{Hydraulic segmentation reduces embolism spreading in xylem tissue}

While the proximity of a studied xylem area to cut conduits seems to be important, the speed of embolism spreading over a certain distance also depends on the vessel dimensions. If each individual vessel would embolise separately (Johnson et al. , 2020), wide and long vessels would show a faster propagation of embolism over a given distance than narrow, short vessels. Spreading of embolism would especially be reduced in xylem with a high degree of hydraulic constrictions, making xylem patches at the distal side of embolised conduits seemingly more resistant to embolism. The four species that showed a reduced embolism resistance in detached leaves as compared to leaves attached to branches, have open vessels running directly from the base of the petiole into the midrib (Table 1, Figure 2). Since the maximum vessel length in petioles of $L$. tulipifera and B. pendula were shorter than the petiole length (Table 1), both species showed relatively small differences in embolism resistance between detached leaves and leaves attached to a stem segment (Fig. 1c, e).

Wide and long vessels in the midrib and secondary veins were found to embolise before the high $3^{\text {rd }}$ to $5^{\text {th }}$ vein orders. This pattern confirms various studies based on the optical method and microCT observations (Brodribb et al. , 2016a, b; Scoffoni et al. , 2017; Klepsch et al. , 2018). However, the observation of local spreading of embolism in minor veins near cut vessels in experiment 2 supports the hypothesis that embolism can spread from pre-existing gas sources, and in minor veins prior to embolism formation in the large vessels of major veins (Fig. 3). This observation suggests that proximity to a gas source is the main driver for embolism spreading, and not the conduit diameter per se. Nevertheless, the limited and short dimensions of minor veins (Chatelet et al. , 2006; Lechthaler et al. , 2019; Hua et al. , 2020) are a plausible explanation for why artificially induced embolism near cuts in minor veins propagate locally, rather than spread across the lamina. Short conduits in minor vein orders may have been selected for in order to maximise leaf hydraulic function over the life span of a leaf. Minor orders of leaf veins are believed to be prone to xylem embolism formation over the life of a leaf due to localized damage caused by insects, pathogens or mechanical damage to the lamina, and because they sustain the most negative pressures (Brodribb et al. , 2010). Considerable restriction to the extent of embolism spread through minor vein orders would ensure a limited impact on 
the hydraulic capacity of a leaf that sustains localised damage to the lamina.

It is also possible that wide and long vessels are more likely connected to a pre-existing embolism than narrow, short conduits, and that large vessels show a higher amount of intervessel pit membrane area than narrow, short vessels. In other words, the reason why large and wide vessels are likely to embolise first, may reflect a difference in the rate of air entry, which is caused by their connectivity to a gas source, and not any inherent difference in embolism resistance per se. We are not aware of an alternative mechanism that would explain why wide conduits are more vulnerable to embolism than narrow ones. Pit membrane thickness, which is strongly associated with embolism resistance (Liet al. , 2016; Kaack et al. , 2019), was found not to be related to conduit diameter (Kotowska et al. , 2020; Wu et al. , 2020).

Vulnerability segmentation may reflect hydraulic segmentation, but not intrinsic differences in embolism resistance per se

The observation that embolism formation in leaves of an individual tree may occur under highly variable (> $1 \mathrm{MPa}$ ) xylem water potentials, suggests that embolism resistance may represent a relative trait that does not capture the absolute, intrinsic embolism resistance of its xylem. It is likely that hydraulic segmentation includes highly reduced conduit dimensions, especially with respect to conduit length and width, with a high number of interconduit end walls over a short stretch of xylem tissue. Conduit end walls have been suggested to hold up embolism spreading at least temporarily, with pit membranes functioning as safety valves and preventing further spreading of embolism due to their tiny pores (Zhang et al., 2017, 2020; Kaacket al. , 2019; Johnson et al. , 2020). Moreover, narrow and short tracheids or fibriform vessels may be more confined than long and wide vessels, with a small interconduit pit membrane area for air entry in narrow tracheids.

Quantifying embolism resistance across the entire xylem pathway could be complicated by measuring artefacts or the proximity to pre-existing embolism, such as cut xylem. In our study, comparison of xylem embolism resistance of leaves attached to long stem segments with embolism resistance of stem xylem based on previous papers (Klepsch et al. , 2018; Zhang et al. , 2018), indicates that leaf xylem of all six species was between 0.5 and $>1 \mathrm{MPa}$ more embolism resistant than stem xylem. Our result for B. pendula was consistent with Klepsch et al. (2018), with leaf xylem being more resistant than stem xylem. Most angiosperms species, however, showed that stem xylem was either more embolism resistant, or equally resistant than leaf xylem (Zhu et al. , 2016; Skelton et al. , 2018; Losso et al. , 2019). Therefore, caution is needed to directly compare absolute values of embolism resistance between organs, since measured values of embolism resistance could be relative estimations only, especially if destructive methods are used and cut-open xylem accelerates embolism spreading.

\section{Embolism spreading may not rely on a fixed pressure difference threshold, but on pressure- driven diffusion}

The observation of embolism spreading under highly variable xylem water potentials in experiment 1 and 2 indicates that embolism formation may not always rely on a certain threshold of the pressure difference between a functional and embolised conduit, as frequently assumed based on the air-seeding hypothesis, and quantitatively estimated by the Young-Laplace equation (Sperry and Tyree, 1988; Choat et al. , 2008). Even if some of our xylem water potential measurements may not be fully accurate due to a pressure gradient heterogeneity (Bouda et al. , 2019), the $>1 \mathrm{MPa}$ difference in $\mathrm{PEP}_{50}$ values between detached leaves and leaves connected to stems provides solid evidence that embolism formation in xylem tissue from the same organ of a species may occur under different xylem water potentials. This finding is not entirely new, and in line with earlier differences in embolism resistance between intact plants and cut plants (Choat et al. , 2010; Torres-Ruiz et al. , 2015; Lamarque et al. , 2018). For instance, a ca. $4 \mathrm{MPa}$ difference in $\mathrm{P}_{50}$ was found for Laurus nobilisbased on microCT observation of cut branches and intact seedlings (Nardini et al. , 2017; Lamarque et al. , 2018). Similar to our results, Skelton et al. (2018) compared the vulnerability curves of cut branches and intact plants based on the optical method forQuercus wislizenii, and found a $1.5 \mathrm{MPa}$ difference in $\mathrm{P}_{50}$ between leaves attached to a long, cut branch, and leaves from an intact plant. The finding that cut plant material can be more vulnerable to embolism spreading than intact plants raises concerns 
about embolism resistance measurements of plant material samples with pre-existing embolism, the possible induction of embolism due to cutting, and the application of the bench dehydration method on cut plant material (Sperry and Tyree, 1988).

If embolism spreading for given species may not rely on a certain pressure threshold, which mechanism does then trigger embolism? We believe that this is a highly important question in our understanding of water transport, even though this question cannot be fully answered based on the available evidence. We speculate that gas diffusion across interconduit pit membranes plays a role in determining the amount of gas dissolved in xylem sap, which may affect embolism nucleation. Although mass flow is theoretically $10^{5}$ times faster than diffusion, gas diffusion in xylem is overall much faster and more common than mass flow. The main reason seems to be that gas diffusion takes place continuously over very large areas, while mass flow requires gas movement through multi-layered, tiny pore constrictions of mesoporous pit membranes (Kaack et al. , 2020). However, the continuous nature of gas diffusion and the high amounts of gas $\left(\mathrm{CO}_{2}\right.$ and $\left.\mathrm{O}_{2}\right)$ surrounding conduits (Spicer and Holbrook, 2005; Teskey et al. , 2008) do not mean that gas concentration in xylem sap is always under equilibrium with gas in embolised conduits, as has frequently been assumed (Hammel 1967; Yang and Tyree, 1992; Wheeleret al. , 2013; Schenk et al. , 2016). Gas solubility of xylem sap is affected by pressure and temperature (Mercury et al. , 2003; Schenk et al. , 2016; Lidon et al. , 2018). Due to a nearly constant atmospheric pressure in a cut-open vessel, but a considerably variable liquid water pressure in sapfilled conduits, the driving force for gas diffusion is the unbalanced, anisobaric situation between embolised conduits and functional conduits. We ignore here the effect of temperature on gas solubility of xylem sap as all experiments were conducted in an air-conditioned lab under similar temperature (Schenk et al. , 2016). Moreover, oversolubility of gas may occur in nanoporous cell walls and mesoporous pit membranes, as is known for nanoconfined environments in general (Pera-Titus et al. , 2009; Hoet al. , 2015; Coasne \& Farrusseng, 2019), which could contribute to concentration gradients of gas dissolved in xylem sap.

A recently embolised intact vessel is not immediately filled with gas under atmospheric pressure, but eventually achieves equilibrium of its gas pressure with the atmosphere, depending on how fast gas is attracted from surrounding gas sources via diffusion. While cut-open vessels are immediately filled with air and reach atmospheric pressure immediately, intact vessels that embolise are initially filled with water vapour (ca. 2.4 $\mathrm{kPa}$ ). It has been modelled that gas diffusion takes from $20 \mathrm{~min}$ to several hours to obtain atmospheric pressure (ca. $101.3 \mathrm{kPa}$ ) in embolised, intact vessels, which may depend on the distance to the nearest gas phase, and the interconduit pit membrane area for gas diffusion (Yang \& Tyree, 1992; Wang et al. , 2015a, b). Although gas diffusion happens across conduit cell walls and pit membranes, the micropores $(<2 \mathrm{~nm})$ in hydrated walls are much smaller than the 5 to $50 \mathrm{~nm}$ dimensions of pit membrane pores (Donaldson et al. , 2019; Kaack et al. , 2019). Therefore, it is reasonable to assume that gas diffusion across hydrated, 200 to $600 \mathrm{~nm}$ thick pit membranes is much faster than across the much thicker layers of secondary cell walls (Yang et al., submitted). Indeed, axial gas diffusion in wood is found to be about one to two orders of magnitude larger than radial diffusion (Sorz \& Hietz, 2006). Moreover, end-wall resistivity of conduits has been suggested to be proportional to lumen resistivity (Sperry et al. , 2005; Hacke et al. , 2006), and conductance of gas increases to the $4^{\text {th }}$ power with conduit diameter or pore diameter according to Hagen-Poiseuille's equation.

Further research is clearly needed to investigate how gas diffusion may contribute to the very high gas solubility of xylem sap (Schenk et al. , 2016), whether oversolubility occurs due to nanoconfined spaces such as pit membranes and cell walls (Pera-Titus et al. , 2009; Hoet al. , 2015; Coasne \& Farrusseng, 2019), how gas-xylem sap interfaces are affected by the dynamic surface tension of xylem sap lipids (Yang et al. , 2020), and how surfactant-coated nanobubbles may contribute to the gas concentration of xylem sap and embolism formation (Schenk et al. , 2015, 2017; Jansen et al. , 2018; Park et al. , 2019).

\section{The optical method and Pneumatron show close similarity in measuring embolism resistance}

We found a strong agreement between the optical method and the Pneumatron, with no significant difference between either $\mathrm{P}_{12}, \mathrm{P}_{50}$ or $\mathrm{P}_{88}$ values obtained from the optical method and the Pneumatron in five deciduous species (Figure 4, Table S2). This finding is in line with the results from Pereira et al. (2020a) for Eucalyptus camaldulensis, and supports the hypothesis that the pneumatic method measures gas extracti- 
on from embolism events in intact vessels (Jansen et al., In press; Yang et al., submitted). The fast and straightforward approach of taking pneumatic methods on small samples such as individual leaves makes the Pneumatron device also suitable for field observations and embolism resistance measurements at the intraspecific and intratree level.

While a high coefficient of determination $\left(R^{2}=0.91\right)$ indicated good agreement between $\mathrm{PEP}_{50}$ and $\mathrm{PAD}_{50}$ values (Fig. 5b), the vulnerability curves with the pneumatic method were less steep than with the optical method for four species studied, except for L. tulipifera. A somewhat weaker, but still significant correlation was found between the $\mathrm{PEP}_{12}$ and $\mathrm{PAD}_{12}$ values based on both methods (Fig. 5a). It is possible that the presence of gas in cut-open vessels affects the process of embolism spreading as discussed above, and this may explain why the percentage of gas discharged by the Pneumatron was slightly higher than the percentage of the cumulative embolised pixels during early stages of dehydration in four out of five species studied (Figure 3, Figure 5A). A similar finding was reported in Pereira et al. (2020a). However, the opposite was found for $P$. avium, which may have slower gas diffusion due to its thick pit membranes as compared to the other species (Kaack et al. , 2020). If intact vessels become embolised, but gas diffusion across pit membranes is slow, atmospheric pressure will not be quickly reached in a recently embolised vessel (Wang et al. , 2015a, b).

The xylem area selected to apply the optical method was deliberately chosen in the upper part of the leaf blade, where vessels in the leaf veins are separated from cut vessels at the petiole end by at least one and most likely various intervessel walls, as shown based on the maximum vessel length in petioles. Since the Pneumatron extracts gas from the petiole end, the tight similarity in embolism resistance between both methods suggests that the gas extracted with the Pneumatron comes from intact, embolised conduits. It is possible that there could be some overlap between vessels from which gas is extracted with the Pneumatron and those that are visualised with the optical method (Fig. 6). Yet, it remains unclear over how many end walls the Pneumatron is able to extract gas. We speculate that this number of end walls depends at least partly on the pit membrane thickness, the complete or partial hydration of the pit membrane, and whether or not porous medium characteristics of interconduit pit membranes change during dehydration, since these would determine gas diffusion considerably (Crombie et al., 1985; Kaacket al., 2019; Zhang et al. , 2020).

\section{Acknowledgements}

We thank Dr. H. Jochen Schenk (California State University, Fullerton) and Lucian Kaack (Ulm University) for valuable comments on an early version of this paper, Dr. Melvin Tyree (Zhejiang Normal University) for useful discussions about pneumatic experiments, two anonymous reviewers for helpful suggestions, and various colleagues from the botanical garden of Ulm University for assistance with sampling. XG, KC, and SJ acknowledge financial support by the German Research Foundation (DFG, project nr. 410768178). SAMM acknowledges the Humboldt Foundation for a postdoctoral fellowship at Ulm University. The authors declare that there is no conflict of interest.

\section{References}

André, J. P., Catesson, A. M., \& Liberman, M. (1999). Characters and origin of vessels with heterogenous structure in leaf and flower abscission zones. Canadian Journal of Botany , 77 (2), 253-261.

André, J. P. (2005). Vascular organization of angiosperms: a new vision . Science Publishers.

Bouda, M., Windt, C. W., McElrone, A. J., \& Brodersen, C. R. (2019). In vivo pressure gradient heterogeneity increases flow contribution of small diameter vessels in grapevine. Nature communications , 10 (1), 1-10.

Bréda, N., Cochard, H., Dreyer, E., \& Granier, A. (1993). Field comparison of transpiration, stomatal conductance and vulnerability to cavitation of Quercus petraea and Quercus robur under water stress. Annales des Sciences Forestières, 50 , 571-582.

Brodersen, C. R., McElrone, A. J., Choat, B., Lee, E. F., Shackel, K. A., \& Matthews, M. A. (2013). In vivo visualizations of drought-induced embolism spread in Vitis vinifera . Plant Physiology , 161 (4), 1820-1829. 
Brodribb, T. J., Feild, T. S., \& Sack, L. (2010). Viewing leaf structure and evolution from a hydraulic perspective. Functional Plant Biology , 37 (6), 488-498.

Brodribb, T. J., Skelton, R. P., McAdam, S. A., Bienaimé, D., Lucani, C. J., \& Marmottant, P. (2016a). Visual quantification of embolism reveals leaf vulnerability to hydraulic failure. New Phytologist, 209 (4), 1403-1409.

Brodribb, T. J., Bienaimé, D., \& Marmottant, P. (2016b). Revealing catastrophic failure of leaf networks under stress. Proceedings of the National Academy of Sciences , 113 (17), 4865-4869.

Brodribb, T. J., Carriqui, M., Delzon, S., \& Lucani, C. (2017). Optical measurement of stem xylem vulnerability. Plant Physiology , 174 (4), 2054-2061.

Chatelet, D. S., Matthews, M. A., \& Rost, T. L. (2006). Xylem structure and connectivity in grapevine (Vitis vinifera) shoots provides a passive mechanism for the spread of bacteria in grape plants. Annals of Botany , 98 (3), 483-494.

Choat, B., Cobb, A. R., \& Jansen, S. (2008). Structure and function of bordered pits: new discoveries and impacts on whole-plant hydraulic function. New Phytologist , 17r7 (3), 608-626.

Choat, B., Drayton, W. M., Brodersen, C., Matthews, M. A., Shackel, K. A., Wada, H., \& Mcelrone, A. J. (2010). Measurement of vulnerability to water stress-induced cavitation in grapevine: a comparison of four techniques applied to a long-vesseled species. Plant, Cell ES Environment, 33 (9), 1502-1512.

Choat, B., Brodersen, C. R., \& McElrone, A. J. (2015). Synchrotron X-ray microtomography of xylem embolism in Sequoia sempervirenssaplings during cycles of drought and recovery. New Phytologist, 205 (3), 1095-1105.

Choat, B., Badel, E., Burlett, R., Delzon, S., Cochard, H., \& Jansen, S. (2016). Noninvasive measurement of vulnerability to drought-induced embolism by X-ray microtomography. Plant Physiology , 170 (1), 273-282.

Coasne, B., \& Farrusseng, D. (2019). Gas oversolubility in nanoconfined liquids: Review and perspectives for adsorbent design. Microporous and Mesoporous Materials, 288, article 109561.

Cochard, H., Badel, E., Herbette, S., Delzon, S., Choat, B., \& Jansen, S. (2013). Methods for measuring plant vulnerability to cavitation: a critical review. Journal of Experimental Botany , 64 (15), 4779-4791.

Cohen, S., Bennink, J., \& Tyree, M. (2003). Air method measurements of apple vessel length distributions with improved apparatus and theory. Journal of Experimental Botany , 54 (389), 1889-1897.

Crombie, D. S., Hipkins, M. F., \& Milburn, J. A. (1985). Gas penetration of pit membranes in the xylem of Rhododendron as the cause of acoustically detectable sap cavitation. Functional Plant Biology , 12 (5), 445-453.

Dixon, H. H., \& Joly, J. (1895). On the ascent of sap.Philosophical Transactions of the Royal Society of London. (B) $186,563-576$.

Domec, J. C., \& Gartner, B. L. (2001). Cavitation and water storage capacity in bole xylem segments of mature and young Douglas-fir trees. Trees , 15 (4), 204-214.

Donaldson, L. A., Singh, A., Raymond, L., Hill, S., \& Schmitt, U. (2019). Extractive distribution in Pseudotsuga menziesii : effects on cell wall porosity in sapwood and heartwood. IAWA Journal , 40 (4), 721-740.

Ellmore, G. S., Zanne, A. E., \& Orians, C. M. (2006). Comparative sectoriality in temperate hardwoods: hydraulics and xylem anatomy. Botanical Journal of the Linnean Society, 150 (1), 61-71.

Espino, S., \& Schenk, H. J. (2009). Hydraulically integrated or modular? Comparing whole-plant-level hydraulic systems between two desert shrub species with different growth forms. New Phytologist, 183 (1), $142-152$. 
Greenidge, K. N. H. (1952). An approach to the study of vessel length in hardwood species. American Journal of Botany, 39 , 570-574.

Hacke, U. G., Sperry, J. S., Wheeler, J. K., \& Castro, L. (2006). Scaling of angiosperm xylem structure with safety and efficiency. Tree Physiology, 26 (6), 689-701.

Hammel, H. T. (1967). Freezing of xylem sap without cavitation. Plant Physiology , 42 (1), 55-66.

Ho, L. N., Schuurman, Y., Farrusseng, D., \& Coasne, B. (2015). Solubility of gases in water confined in nanoporous materials: ZSM-5, MCM-41, and MIL-100. The Journal of Physical Chemistry C , 119 (37), 21547-21554.

Hochberg, U., Albuquerque, C., Rachmilevitch, S., Cochard, H., David-Schwartz, R., Brodersen, C. R., .. \& \& Windt, C. W. (2016). Grapevine petioles are more sensitive to drought induced embolism than stems: evidence from in vivo MRI and microcomputed tomography observations of hydraulic vulnerability segmentation. Plant, Cell \& Environment, 39 (9), 1886-1894.

Hochberg, U., Windt, C. W., Ponomarenko, A., Zhang, Y. J., Gersony, J., Rockwell, F. E., \& Holbrook, N. M. (2017). Stomatal closure, basal leaf embolism, and shedding protect the hydraulic integrity of grape stems. Plant Physiology, 174 (2), 764-775.

Hua, L., He, P., Goldstein, G., Liu, H., Yin, D., Zhu, S., \& Ye, Q. (2020). Linking vein properties to leaf biomechanics across 58 woody species from a subtropical forest. Plant Biology , 22 (2), 212-220.

Jansen, S., \& Schenk, H. J. (2015). On the ascent of sap in the presence of bubbles. American Journal of Botany , 102 (10), 1561-1563.

Jansen, S., Klepsch, M., Li, S., Kotowska, M. M., Schiele, S., Zhang, Y., \& Schenk, H. J. (2018). Challenges in understanding air-seeding in angiosperm xylem. Acta Horticulturae, 1222 , 13-20.

Jansen, S., Guan, X., Kaack, L., Trabi, C., Miranda, M. T., Ribeiro, R. V., \& Pereira, L. (In press) The Pneumatron estimates xylem embolism resistance in angiosperms based on gas diffusion kinetics: a minireview. Acta Horticulturae .

Johnson, K. M., Brodersen, C., Carins-Murphy, M. R., Choat, B., \& Brodribb, T. J. (2020). Xylem embolism spreads by single-conduit events in three dry forest angiosperm stems. Plant Physiology , 184 (1), 212-222.

Kaack, L., Altaner, C. M., Carmesin, C., Diaz, A., Holler, M., Kranz, C., ... Jansen, S. (2019). Function and three-dimensional structure of intervessel pit membranes in angiosperms: a review. IAWA Journal, 40 (4), 673-702.

Kaack, L., Weber, M., Isasa, E., Karimi, Z., Li, S., Pereira, L., . . \& Schmidt, V. (2020). Pore constrictions in intervessel pit membranes reduce the risk of embolism spreading in angiosperm xylem. bioRxiv .

Kitin, P. B., Fujii, T., Abe, H., \& Funada, R. (2004). Anatomy of the vessel network within and between tree rings of Fraxinus lanuginosa (Oleaceae). American Journal of Botany , 91 (6), 779-788.

Klepsch, M., Zhang, Y., Kotowska, M. M., Lamarque, L. J., Nolf, M., Schuldt, B., .. \& \& Scoffoni, C. (2018). Is xylem of angiosperm leaves less resistant to embolism than branches? Insights from microCT, hydraulics, and anatomy. Journal of Experimental Botany , 69 (22), 5611-5623.

Kotowska, M. M., Thom, R., Zhang, Y., Schenk, H. J., \& Jansen, S. (2020). Within-tree variability and sample storage effects of bordered pit membranes in xylem of Acer pseudoplatanus. Trees , 34 (1), 61-71.

Lamarque, L. J., Corso, D., Torres-Ruiz, J. M., Badel, E., Brodribb, T. J., Burlett, R., .. \& Jansen, S. (2018). An inconvenient truth about xylem resistance to embolism in the model species for refilling Laurus nobilis L. Annals of Forest Science, 75 (3), article 88. 
Lechthaler, S., Colangeli, P., Gazzabin, M., \& Anfodillo, T. (2019). Axial anatomy of the leaf midrib provides new insights into the hydraulic architecture and cavitation patterns of Acer pseudoplatanus leaves. Journal of Experimental Botany, 70 (21), 6195-6201.

Levionnois, S., Ziegler, C., Jansen, S., Calvet, E., Coste, S., Stahl, C., .. \& Heuret, P. (2020). Vulnerability and hydraulic segmentations at the stem-leaf transition: coordination across Neotropical trees. New Phytologist, 228 (2), 512-524.

Li, S., Lens, F., Espino, S., Karimi, Z., Klepsch, M., Schenk, H. J., . . \& Jansen, S. (2016). Intervessel pit membrane thickness as a key determinant of embolism resistance in angiosperm xylem. IAWA Journal, 37 (2), 152-171.

Lidon, P., Marker, S. C., Wilson, J. J., Williams, R. M., Zipfel, W. R., \& Stroock, A. D. (2018). Enhanced oxygen solubility in metastable water under tension. Langmuir , 34 (40), 12017-12024.

Loepfe, L., Martinez-Vilalta, J., Pinol, J., \& Mencuccini, M. (2007). The relevance of xylem network structure for plant hydraulic efficiency and safety. Journal of Theoretical Biology , 247 (4), 788-803.

Losso, A., Bar, A., Damon, B., Dullin, C., Ganthaler, A., Petruzzellis, F., .. \& Beikircher, B. (2019). Insights from in vivo micro-CT analysis: testing the hydraulic vulnerability segmentation in Acer pseudoplatanus and Fagus sylvatica seedlings. New Phytologist, 221 (4), 1831-1842.

Martin-StPaul, N., Delzon, S., \& Cochard, H. (2017). Plant resistance to drought depends on timely stomatal closure. Ecology Letters , 20 (11), 1437-1447.

Morris, H., Brodersen, C., Schwarze, F. W., \& Jansen, S. (2016). The parenchyma of secondary xylem and its critical role in tree defense against fungal decay in relation to the CODIT model. Frontiers in Plant Science, 7 , article 1665 .

Mercury, L., Azaroual, M., Zeyen, H., \& Tardy, Y. (2003). Thermodynamic properties of solutions in metastable systems under negative or positive pressures. Geochimica et Cosmochimica Acta , 67 (10), 17691785 .

Nardini, A., Savi, T., Losso, A., Petit, G., Pacile, S., Tromba, G., . . \& Salleo, S. (2017). X-ray microtomography observations of xylem embolism in stems of Laurus nobilis are consistent with hydraulic measurements of percentage loss of conductance. New Phytologist, 213 (3), 1068-1075.

Oliveira, R. S., Costa, F. R., van Baalen, E., de Jonge, A., Bittencourt, P. R., Almanza, Y., .. \& Guimaraes, Z. T. (2019). Embolism resistance drives the distribution of Amazonian rainforest tree species along hydrotopographic gradients. New Phytologist, 221 (3), 1457-1465.

Oskolski, A. A., \& Jansen, S. (2009). Distribution of scalariform and simple perforation plates within the vessel network in secondary xylem of Araliaceae and its implications for wood evolution. Plant Systematics and Evolution, 278 (1-2), 43-51.

Pammenter, N. V., \& Van der Willigen, C. (1998). A mathematical and statistical analysis of the curves illustrating vulnerability of xylem to cavitation. Tree Physiology , 18 (8-9), 589-593.

Pan, R., Geng, J., Cai, J., \& Tyree, M. T. (2015). A comparison of two methods for measuring vessel length in woody plants. Plant, Cell \& Environment, 38 (12), 2519-2526.

Park, J., Go, T., Ryu, J., \& Lee, S. J. (2019). Air spreading through wetted cellulose membranes: Implications for the safety function of hydraulic valves in plants. Physical Review E , 100 (3), article 032409.

Pera-Titus, M., El-Chahal, R., Rakotovao, V., Daniel, C., Miachon, S., \& Dalmon, J. A. (2009). Direct volumetric measurement of gas oversolubility in Nanoliquids: beyond Henry's law. ChemPhysChem , 10 (12), 2082-2089. 
Pereira, L., Bittencourt, P. R., Oliveira, R. S., Junior, M. B., Barros, F. V., Ribeiro, R. V., \& Mazzafera, P. (2016). Plant pneumatics: stem air flow is related to embolism-new perspectives on methods in plant hydraulics. New Phytologist, 211 (1), 357-370.

Pereira, L., Bittencourt, P. R., Pacheco, V. S., Miranda, M. T., Zhang, Y., Oliveira, R. S., .. \& Rowland, L. (2020a). The Pneumatron: An automated pneumatic apparatus for estimating xylem vulnerability to embolism at high temporal resolution. Plant, Cell \& Environment , 43 (1), 131-142.

Pereira, L., Miranda, M. T., Pires, G. S., Pacheco, V. S., Guan, X., Kaack, L., .. \& Ribeiro, R. V. (2020b). A semi-automated method for measuring xylem vessel length distribution. Theoretical and Experimental Plant Physiology , 32, 331-340.

Salleo, S., Gullo, M. L., \& Siracusano, L. (1984). Distribution of vessel ends in stems of some diffuse-and ring-porous trees: the nodal regions as 'safety zones' of the water conducting system. Annals of Botany , 54 (4), 543-552.

Sano, Y., Morris, H., Shimada, H., Ronse De Craene, L. P., \& Jansen, S. (2011). Anatomical features associated with water transport in imperforate tracheary elements of vessel-bearing angiosperms. Annals of Botany , 107 (6), 953-964.

Schenk, H. J., Steppe, K., \& Jansen, S. (2015). Nanobubbles: a new paradigm for air-seeding in xylem. Trends in Plant Science, 20 (4), 199-205.

Schenk, H. J., Espino, S., Visser, A., \& Esser, B. K. (2016). Dissolved atmospheric gas in xylem sap measured with membrane inlet mass spectrometry. Plant, Cell $\&$ Environment , 39 (4), 944-950.

Schenk, H. J., Espino, S., Romo, D. M., Nima, N., Do, A. Y., Michaud, J. M., . . \& \& Jansen, S. (2017). Xylem surfactants introduce a new element to the cohesion-tension theory. Plant Physiology, 173 (2), 1177-1196.

Schindelin, J., Arganda-Carreras, I., Frise, E., Kaynig, V., Longair, M., Pietzsch, T., .. \& \& Tinevez, J. Y. (2012). Fiji: an open-source platform for biological-image analysis. Nature Methods , 9 (7), 676-682.

Scoffoni, C., Albuquerque, C., Brodersen, C. R., Townes, S. V., John, G. P., Cochard, H., .. \& \& Sack, L. (2017). Leaf vein xylem conduit diameter influences susceptibility to embolism and hydraulic decline. New Phytologist , 213 (3), 1076-1092.

Skelton, R. P., Dawson, T. E., Thompson, S. E., Shen, Y., Weitz, A. P., \& Ackerly, D. (2018). Low vulnerability to xylem embolism in leaves and stems of North American oaks. Plant Physiology , 177 (3), 1066-1077.

Sorz, J., \& Hietz, P. (2006). Gas diffusion through wood: implications for oxygen supply. Trees , 20 (1), $34-41$.

Sperry, J. S., \& Tyree, M. T. (1988). Mechanism of water stress-induced xylem embolism. Plant Physiology , 88 (3), 581-587.

Sperry, J. S., Hacke, U. G., \& Wheeler, J. K. (2005). Comparative analysis of end wall resistivity in xylem conduits. Plant, Cell \& Environment, 28 (4), 456-465.

Spicer, R., \& Holbrook, N. M. (2005). Within-stem oxygen concentration and sap flow in four temperate tree species: does long-lived xylem parenchyma experience hypoxia? Plant, Cell ES Environment, 28 (2), 192-201.

Teskey, R. O., Saveyn, A., Steppe, K., \& McGuire, M. A. (2008). Origin, fate and significance of $\mathrm{CO}_{2}$ in tree stems. New Phytologist, 177 (1), 17-32.

Tixier, A., Herbette, S., Jansen, S., Capron, M., Tordjeman, P., Cochard, H., \& Badel, E. (2014). Modelling the mechanical behaviour of pit membranes in bordered pits with respect to cavitation resistance in angiosperms. Annals of Botany , 114 (2), 325-334. 
Torres-Ruiz, J. M., Jansen, S., Choat, B., McElrone, A. J., Cochard, H., Brodribb, T. J., . . \& Li, S. (2015). Direct X-ray microtomography observation confirms the induction of embolism upon xylem cutting under tension. Plant Physiology , 167 (1), 40-43.

Tyree, M. T., \& Ewers, F. W. (1991). The hydraulic architecture of trees and other woody plants. New Phytologist , 119 (3), 345-360.

Tyree, M. T., Alexander, J., \& Machado, J. L. (1992). Loss of hydraulic conductivity due to water stress in intact juveniles of Quercus rubra and Populus deltoides . Tree Physiology, 10 (4), 411-415.

Wang, R., Zhang, L., Zhang, S., Cai, J., \& Tyree, M. T. (2014). Water relations of Robinia pseudoacacia L.: do vessels cavitate and refill diurnally or are R-shaped curves invalid in Robinia ? Plant, Cell Es Environment , 37 (12), 2667-2678.

Wang, Y., Pan, R., \& Tyree, M. T. (2015a). Studies on the tempo of bubble formation in recently cavitated vessels: a model to predict the pressure of air bubbles. Plant Physiology , 168 (2), 521-531.

Wang, Y., Liu, J., \& Tyree, M. T. (2015b). Stem hydraulic conductivity depends on the pressure at which it is measured and how this dependence can be used to assess the tempo of bubble pressurization in recently cavitated vessels. Plant physiology , 169 (4), 2597-2607.

Wason, J. W., Anstreicher, K. S., Stephansky, N., Huggett, B. A., \& Brodersen, C. R. (2018). Hydraulic safety margins and air-seeding thresholds in roots, trunks, branches and petioles of four northern hardwood trees. New Phytologist, 219 (1), 77-88.

Wheeler, J. K., Huggett, B. A., Tofte, A. N., Rockwell, F. E., \& Holbrook, N. M. (2013). Cutting xylem under tension or supersaturated with gas can generate PLC and the appearance of rapid recovery from embolism. Plant, Cell \& Environment, 36 (11), 1938-1949.

Wolfe, B. T., Sperry, J. S., \& Kursar, T. A. (2016). Does leaf shedding protect stems from cavitation during seasonal droughts? A test of the hydraulic fuse hypothesis. New Phytologist , 212 (4), 1007-1018.

Wu M., Zhang Y., Oya T., Marcati C.R., Pereira L., Jansen S.(2020) Root xylem in three woody angiosperm species is not more vulnerable to embolism than stem xylem. Plant and Soil, $450,479-495$.

Yang, S., \& Tyree, M. T. (1992). A theoretical model of hydraulic conductivity recovery from embolism with comparison to experimental data on Acer saccharum . Plant, Cell 83 Environment ,15 (6), 633-643.

Yang, J., M Michaud, J., Jansen, S., Schenk, H. J., \& Zuo, Y. Y. (2020). Dynamic surface tension of xylem sap lipids. Tree Physiology , 40 (4), 433-444.

Zhang, Y., Klepsch, M., \& Jansen, S. (2017). Bordered pits in xylem of vesselless angiosperms and their possible misinterpretation as perforation plates. Plant, Cell $\& 3$ Environment , 40 (10), 2133-2146.

Zhang, Y., Lamarque, L. J., Torres-Ruiz, J. M., Schuldt, B., Karimi, Z., Li, S., .. \& \&elzon, S. (2018). Testing the plant pneumatic method to estimate xylem embolism resistance in stems of temperate trees. Tree Physiology , 38 (7), 1016-1025.

Zhang, Y., Carmesin, C., Kaack, L., Klepsch, M. M., Kotowska, M., Matei, T., .. \& \& Jansen, S. (2020). High porosity with tiny pore constrictions and unbending pathways characterize the 3D structure of intervessel pit membranes in angiosperm xylem. Plant, Cell \& Environment , 43 (1), 116-130.

Zhu, S. D., Song, J. J., Li, R. H., \& Ye, Q. (2013). Plant hydraulics and photosynthesis of 34 woody species from different successional stages of subtropical forests. Plant, Cell $\& 3$ Environment, 36 (4), 879-891.

Zhu, S. D., Liu, H., Xu, Q. Y., Cao, K. F., \& Ye, Q. (2016). Are leaves more vulnerable to cavitation than branches? Functional Ecology, 30 (11), 1740-1744.

Zimmermann, M. H. (1983). Xylem structure and the ascent of sap. Springer Science \& Business Media. 


\section{Tables}

Table 1. Overview of the petiole length and vessel length dimensions for six temperate tree species studied. Maximum vessel length measurements were based on air injection, while the average length of vessels starting at the basipetal end of the petiole was estimated with a Pneumatron. Values indicate mean +- standard deviation $(\mathrm{n}=5$ or 6$)$.

\begin{tabular}{llll}
\hline & Petiole length $(\mathrm{cm})$ & Max. vessel length at the petiole end $(\mathrm{cm})$ & Average vessel length at the base of the $\mathrm{p}$ \\
\hline B. pendula & $2.03 \pm 0.48$ & $1.07 \pm 0.26$ & $0.83 \pm 0.09$ \\
C. betulus & $1.37 \pm 0.13$ & $1.44 \pm 0.30$ & $0.96 \pm 0.12$ \\
F. sylvatica & $1.23 \pm 0.06$ & $3.41 \pm 0.27$ & $0.66 \pm 0.43$ \\
L. tulipifera & $8.68 \pm 1.39$ & $8.19 \pm 1.47$ & $3.52 \pm 1.17$ \\
P. avium & $3.53 \pm 1.00$ & $5.47 \pm 0.83$ & $/$ \\
Q. petraea & $1.17 \pm 0.21$ & $8.02 \pm 1.33$ & $5.1 \pm 0.90$ \\
\hline
\end{tabular}

\section{Figure legends}

Figure 1. Leaf xylem vulnerability curves of six deciduous species using the optical method applied to different samples: a detached leaf (red), leaf attached to a short stem segment (blue), and to a long branch (green). For each sample type, four to five replicates were measured (pale colours). Bright lines are regression lines for each type of sample, and grey lines indicate $95 \%$ confidential intervals. $\mathrm{PEP}_{50}$ values $(\mathrm{MPa})$ of the three types of samples were presented with mean \pm standard deviation $(\mathrm{n}=4$ or 5$)$, with different small letters showing significant difference between each sample type $(P<0.05)$. Note that the curve fitting may give the impression that embolism occurs at positive xylem pressures, while the raw data make clear that embolism spreading never occurs at a xylem water potential that is less negative than -0.1 MPa.

Figure 2 . Relationship between the segmentation index and the difference in $\mathrm{PEP}_{12}$ (red), $\mathrm{PEP}_{50}$ (yellow) and $\mathrm{PEP}_{88}$ (green) values between detached leaves and leaves attached to a short stem segment. A segmentation index was defined as the maximum vessel length divided by the petiole length, with vessels running into the leaf blade when the value is $>1$, and the longest vessels ending before the leaf blade when the value is $<1$.

Figure 3. Maps of embolism events between an intact leaf (A-F) and a leaf with cuts in minor veins (G-L) of Quercus petraea. The cut and intact leaf pair were attached to the same long branch, with images taken for both leaves after a certain desiccation time (shown on the left). White solid lines represent artificial cuts of the $3^{\text {rd }}$ or $4^{\text {th }}$ veins, and white arrows point at embolism in minor veins near the cuts, which happened much earlier than embolism in major veins.

Figure 4 . Xylem vulnerability curves of leaves of five deciduous species based on the optical and pneumatic method. Both methods were applied to the same detached leaf. Pale and bright red lines represent raw data and regression lines of the optical method, respectively. Blue lines represent raw data (pale blue) and regression lines (bright blue) based on the pneumatic method. For each species, four leaves were tested, and $\mathrm{P}_{50}$ values $(\mathrm{MPa})$ of both methods were presented.

Figure 5 . Correlation between paired values of $\mathrm{P}_{12}(\mathrm{a}), \mathrm{P}_{50}(\mathrm{~b})$, and $\mathrm{P}_{88}$ (c) as measured with the optical and pneumatic method. Each dot represents a single leaf, solid black lines indicate the regression line, blue dashed lines indicate the 95\% confidence intervals, and black dashed lines indicate the 1:1 line.

Figure 6 . Illustration of the optical method and pneumatic measurements on detached leaves and possible embolism spreading during dehydration after 5 and 10 hours. The Pneumatron was connected at the basipetal end of the petiole, while the leaf area scanned with the optical method (pale yellow square) is separated from the cut-open vessels (white) by at least several end walls. (a) The initial status when leaves were fresh, and all intact vessels were water-filled (blue). (b, c) Embolism spreads to various intact vessels (green), from 
which gas is extracted with the Pneumatron, and which are observed with the optical method when these are within the scanned leaf area. It is assumed here that the Pneumatron extracts gas across two end walls. Arrows indicate gas diffusion through intervessel walls towards the Pneumatron. Embolism formation in vessels only captured with the optical method are coloured yellow. Adapted from Jansen et al. (In press).

\section{Hosted file}

Figures.pdf available at https://authorea.com/users/349270/articles/493522-no-gas-source-noproblem-proximity-to-pre-existing-embolism-and-segmentation-affect-embolism-spreadingin-angiosperm-xylem-by-gas-diffusion 